\title{
Recent trends in intensity-modulated radiation therapy use in Korea
}

\author{
Seung Jae Huh", Won Park², Do Ho Choi ${ }^{2}$ \\ ${ }^{1}$ Healthcare Review and Assessment Committee, Health Insurance Review and Assessment Service, Seoul, Korea \\ ${ }^{2}$ Department of Radiation Oncology, Samsung Medical Center, Sungkyunkwan University School of Medicine, Seoul, Korea
}

Purpose: We aimed to analyze the trend in intensity-modulated radiation therapy (IMRT) use in Korea from 2011 to 2018.

Materials and Methods: We collected data from the Health and Insurance Review and Assessment Service (HIRA) big data based on the National Health Insurance Service claims and reimbursements records using primary treatment planning codes (HD 041) for IMRT from 2011 to 2018. We analyzed the changing patterns in clinical application to specific tumor sites and regional differences in IMRT utilization.

Results: The use of IMRT has exhibited an 18-fold steep rise from 1,921 patients in 2011 to 34,759 in 2018. With regard to IMRT in $2018,70 \%$ of patients $(24,248 / 34,759)$ were treated in metropolitan areas (Seoul, Incheon, and Gyeonggi Province). IMRT was most commonly used to treat breast, lung, and prostate cancers in 2018. Among these, the use of IMRT for breast cancer shows the most remarkable increase from 2016 when the National Health Insurance began to cover IMRT for all solid tumors.

Conclusion: The use of IMRT is steadily increasing to treat cancer and is concentrated in metropolitan areas.

Keywords: Intensity-modulated radiotherapy, Korea

\section{Introduction}

In 2016, there were 203,129 new cancers in Korea except thyroid cancer, with the highest incidence in the order of stomach, colon, lung, breast and liver cancer. For men, the incidence rate of gastric cancer, colorectal cancer, and liver cancer has decreased, but prostate cancer increased steadily by $13 \%$ until 2009, but no longer increasing. In women, stomach cancer, colon cancer, liver cancer and cervical cancer are continuously decreasing, but breast cancer is steadily increasing [1].

To date, radiation therapy technology has steadily improved to reduce adjacent normal organ side effects and improve therapeutic effects in tumors. Radiation therapy has evolved from conventional two-dimensional therapy to three-dimensional conformal therapy (3D-CRT). Recently, radiotherapy technologies using intensity-modulated radiation therapy (IMRT) have been applied in most cancers. The use of IMRT to treat cancer is steadily increasing in Korea $[2,3]$. According to a recent report based on the big data of the Health and Insurance Review and Assessment Service (HIRA), IMRT was the second-most common radiotherapy modality following 3D-CRT, and the IMRT utilization rate in Korea was $23 \%$ in 2016 [2].

Received 31 October 2019, Revised 25 November 2019, Accepted 12 December 2019.

Correspondence: Seung Jae Huh, Healthcare Review and Assessment Committee, Health Insurance Review and Assessment Service, 267 Hyoryeongno, Seocho-gu, Seoul 06653, Korea. Tel: +82-2-3410-2616, E-mail: sjhuh5201@gmail.com (http:// orcid.org/0000-0002-3635-8253)

(c) This is an Open Access article distributed under the terms of the Creative Commons Attribution Non-Commercial License (http://creativecommons.org/ licenses/by-nc/4.0/) which permits unrestricted non-commercial use, distribution, and reproduction in any medium, provided the original work is properly cited.

www.e-roj.org 
Table 1. Number of patients who received primary treatment planning for IMRT by cancer site from 2011 to 2018

\begin{tabular}{lrrrrrrrr}
\hline & 2011 & 2012 & 2013 & 2014 & 2015 & 2016 & 2017 & 2018 \\
\hline Breast & 45 & 87 & 96 & 69 & 400 & 1,362 & 2,526 & 6,674 \\
Lung & 124 & 258 & 197 & 244 & 722 & 2,414 & 3,297 & 4,688 \\
Prostate & 414 & 1,378 & 1,539 & 1,519 & 1,649 & 2,228 & 2,653 & 3,236 \\
Gastro-intestine & 89 & 84 & 72 & 72 & 206 & 1,701 & 2,201 & 2,716 \\
Hepatobiliary and pancreas & 60 & 112 & 95 & 77 & 391 & 1,330 & 1,620 & 2,054 \\
Head and neck & 668 & 2,181 & 2,343 & 2,414 & 2,423 & 2,348 & 2,259 & 2,001 \\
Cervix & 42 & 81 & 80 & 82 & 239 & 721 & 852 & 1,145 \\
Central nervous system & 134 & 448 & 582 & 707 & 719 & 828 & 912 & 1,103 \\
Others & 297 & 787 & 871 & 1,055 & 2,085 & 6,224 & 7,573 & 11,142 \\
Total & 1,873 & 5,416 & 5,875 & 6,239 & 8,834 & 19,156 & 23,893 & 34,759 \\
\hline
\end{tabular}

IMRT, intensity-modulated radiation therapy.

Until now, there have been few reports of IMRT use since the first implementation of IMRT in 2001 in Korea [2-4]. Moreover, there has been no report regarding the utilization of IMRT for specific cancer sites. Herein, we analyzed the current status of and changing patterns in IMRT use for specific cancer sites from 2011 to 2018.

\section{Materials and Methods}

We collected data from the HIRA based on the National Health Insurance Service claims and reimbursements records using primary treatment planning codes for IMRT (HD 041) from 2011 to 2018 [5]. The total amount of IMRT use was tallied based on the year of the insurance audit of the HIRA data. We analyzed changing patterns in clinical implementation according to the cancer site by year. We also investigated IMRT utilization by administrative district in 2018. Additionally, we used the status of radiotherapy facilities and machines at the end of 2018 [6].

\section{Results}

The total numbers of patients who received IMRT in 2011, 2013, 2015, and 2018 were 1,921, 5,992, 9,091, and 34,759, respectively (Table 1 ). The use of IMRT has exhibited an 18.6-fold steep rise from 2011 to 2018. Three cancers most frequently treated with IMRT in 2011 were head and neck, prostate, and central nervous system cancers, but in 2018, these changed to breast, lung, and prostate cancers. In 2018, compared to 2011, IMRT for breast cancer increased 148.3-fold in 2018, and the frequencies of IMRT in lung, hepatobiliary, and gastrointestinal cancers increased 37.8-, 34.2-, and 30.5-fold, respectively. Since the application of IMRT health insurance coverage in 2015, IMRT use increased dramatically in 2016 for most cancers (Fig. 1).

In 2018, 70\% of new patients who received IMRT $(24,248 / 34,759)$ were treated in metropolitan area such as Seoul, Incheon, and Gyeonggi areas. However, only 6.7\% of patients received IMRT in areas that were not metropolitan, such as Gangwon, Chungbuk, Jeonbuk, and Jeju. In 2018, there were 91 radiation treatment facilities and 205 radiation treatment machines nationwide [6]. In 2018, the average number of IMRT patients administered per facility was 382.0, and the average number of IMRT patients administered per machine was 169.6 (Table 2). In Seoul, Incheon, and Gyeonggi areas, the number of IMRT patients administered per radiation therapy facility was 499.9, which was higher than the average, and the number of IMRT patients administered per machine was 190.2, which was also higher than the average. In areas that were not metropolitan, such as Gangwon, Chungbuk, Jeonbuk, and Jeju, the number of IMRTs administered per radiation therapy facility was 298.1 , which was lower than the average, but the number of IMRT patients administered per machine was 174.5, which was slightly higher than the average.

\section{Discussion and Conclusion}

The use of radiotherapy in cancer patients has increased steadily from 25\% in 2011 to 30\% in 2015, and the use of IMRT is also rapidly increasing in Korea $[2,3]$. The current study showed that IMRT use has been increasing rapidly, showing an 18-fold steep rise from 1,921 patients in 2011 to 34,759 in 2018. Since IMRT was first performed in 2001 in 


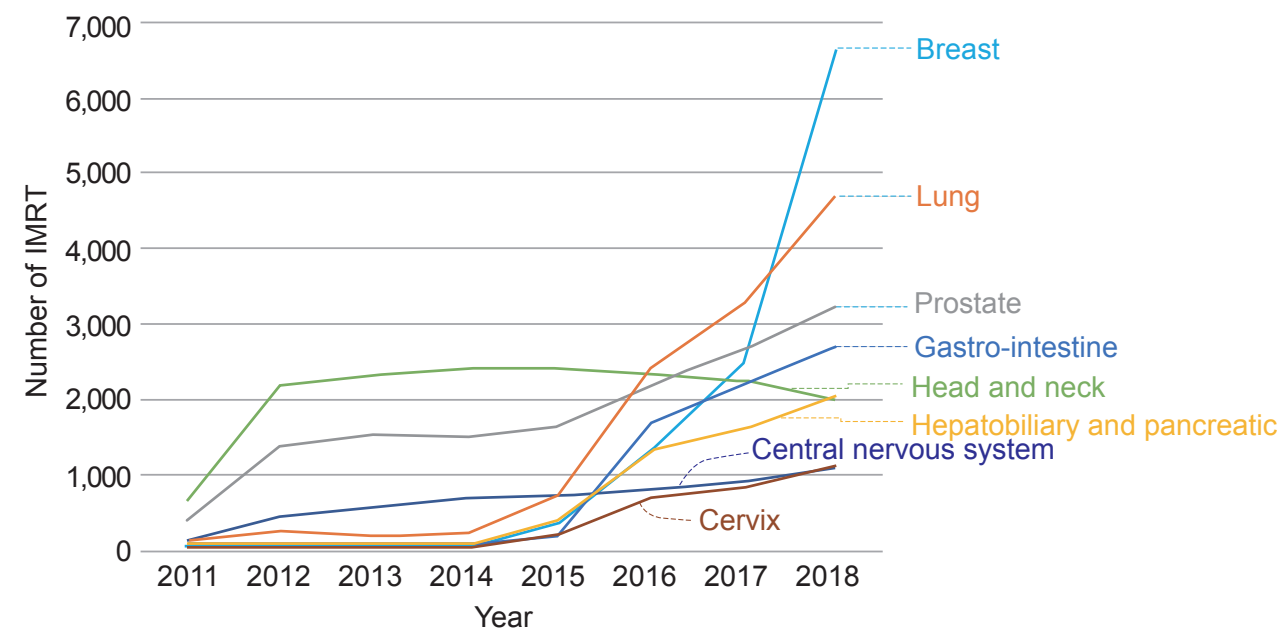

Fig. 1. Use of intensity-modulated radiation therapy (IMRT) showed a remarkable increase from 2016 since the National Health Insurance expanded reimbursement for IMRT to include all solid tumors in 2015.

Table 2. Number of IMRT patients administered per radiation facility and machine per year according to area in 2018

\begin{tabular}{lccccc}
\hline \multicolumn{1}{c}{ Area } & $\begin{array}{c}\text { Number of IMRT } \\
\text { patients }\end{array}$ & $\begin{array}{c}\text { Number of radiation } \\
\text { facilities }\end{array}$ & $\begin{array}{c}\text { Number of IMRT } \\
\text { patients per facility }\end{array}$ & $\begin{array}{c}\text { Radiation treatment } \\
\text { machines }\end{array}$ & $\begin{array}{c}\text { Number of IMRT } \\
\text { patients per } \\
\text { machine }\end{array}$ \\
\hline Seoul & 16,903 & 26 & 650.1 & 84 & 201.2 \\
Gyeonggi, Incheon & 7,345 & 21 & 349.8 & 41 & 179.1 \\
Gyeongnam, Busan, Ulsan & 3,079 & 12 & 256.6 & 26 & 118.4 \\
Gyeongbuk, Daegu & 2,132 & 11 & 193.8 & 18 & 118.4 \\
Chungnam, Daejeon & 1,661 & 8 & 207.6 & 13 & 127.8 \\
Jeonnam, Gwangju & 1,319 & 3 & 439.7 & 8 & 164.9 \\
Gangwon & 843 & 4 & 210.8 & 6 & 140.5 \\
Chungbuk & 688 & 1 & 688.0 & 2 & 344.0 \\
Jeonbuk & 604 & 3 & 201.3 & 5 & 120.8 \\
Jeju & 185 & 2 & 92.5 & 2 & 92.5 \\
Total number & 34,759 & 91 & - & 205 & - \\
\hline
\end{tabular}

IMRT, intensity-modulated radiation therapy.

Korea, implementation of IMRT has been partially covered by national health insurance for specific cancer sites (head and neck, brain, prostate, spinal cancers and re-irradiation cases) from 2011, and since July 2015, the coverage has expanded to include almost all cancers [4]. An abrupt increase from 2015, despite some controversy regarding the cost-effectiveness of IMRT, larger skin dose, and risk of radiation-induced secondary cancers, may have been due to the health insurance coverage and recent advancement in therapeutic machines for IMRT $[2,3,7]$.

The current study showed that the three cancers most frequently treated with IMRT in 2011 were head and neck, prostate, and central nervous system cancers, but in 2018, these were breast, lung, and prostate cancers (Table 1). Among these, the use of IMRT for breast cancer showed the most remarkable increase. In Korea, the use of IMRT to treat breast cancer is steadily increasing to spare organs at risk, particularly the heart, in patients with left breast cancer and for hypofractionated whole-breast irradiation since 2015. According to the UK IMRT survey report in 2008, there is considerable variety in the definition of IMRT for breast cancer, with some studies using an inverse-planned technique with multiple gantry angles and others using so-called simple IMRT, consisting of a forward-planned field-within-field 
technique. This report showed that for breast cancer, forwardplanned IMRT is prominently used instead of inverse-planned IMRT (5,517 vs. 56 patients) [8]. Despite the demonstrated benefit of modulating the intensity of the treatment field, there remains uncertainty regarding when the less expensive simple IMRT or 3D-CRT may be adequate and when the more resource-intensive, inverse-planned, high-cost IMRT is needed. In its 'Choosing Wisely' campaign, the American Society for Radiation Oncology (ASTRO) recommended that IMRT should not be routinely used to deliver whole-breast radiation as part of breast conservation therapy. Although IMRT may be beneficial in selected cases involving unusual anatomy, no studies have yet demonstrated that its routine use provides a significant clinical advantage [9]. According to the 2018 ASTRO evidence-based guideline for the whole breast radiation therapy, three-dimensional conformal treatment planning (3D-CRT) with a 'field-in-field' technique is strongly recommended as the initial treatment planning approach with $100 \%$ consensus, However, the guideline comments nothing about the role of IMRT [10].

IMRT utilization is increasing worldwide; however, there is a difference in the use of IMRT across individual treatment centers and by country $[8,11]$. Shumway et al. [11] reported a 4-fold variation in IMRT use among centers across the State of Michigan ranging from 23\% in the lowest-use center to 96\% in the highest-use center in 2012. The utilization rate of IMRT in radiation therapy in Japan was 15\% in 2017, while it was 23\% in Korea in 2016 [2,12]. In Japan, IMRT is only reimbursed by the national health insurance system when the following personnel are present: (1) two full-time radiation oncologists and a radiotherapy technician, each with more than 5 years of radiotherapy experience; and (2) an individual responsible solely for precision control of the radiotherapy devices, irradiation plan verification, and assistance with the irradiation plan (e.g., a radiotherapy or other technician) [13]. According to the Japanese Society for Radiation Oncology database report of 2018, IMRT was mostly used to treat prostate, head and neck, and central nerve system tumors in Japan [14]. On the other hand, in Korea, IMRT was most commonly used to treat breast, lung, and prostate cancers in 2018.

With regard to IMRT in 2018, 70\% of new patients $(24,248 / 34,759)$ were treated in metropolitan areas. The main reason for the need for medical travel to metropolitan areas among patients receiving radiotherapy was the concentration of facilities in the capital area and Korea's advanced public transport system, including the high-speed railway line [15].

Our study has several limitations. The current study only included data of the insured patients for primary treatment planning for IMRT, excluding data of secondary planning for boost treatment, and the number of uninsured or foreign patients could not be recorded. Therefore, the actual number of patients who received IMRT is expected to be higher than the reported number. Despite these limitations, we think that this study provides the latest trends in the use of IMRT for specific cancer sites. In conclusion, the use of IMRT has been steadily increasing to treat cancer since 2011 and shows a metropolitan area concentration. Currently, IMRT is most commonly used to treat breast, lung, and prostate cancers.

In summary, it is necessary to redefine the clinical application of IMRT on the basis of prospective clinical studies that can prove clinical benefits of IMRT over 3D-CRT in the treatment of cancer patients. In addition, reviewing the cost-benefits will help to establish the basis for the clinical application of IMRT.

\section{Conflict of Interest}

No potential conflict of interest relevant to this article was reported.

\section{Acknowledgments}

We are grateful to the Health and Insurance Review and Assessment Service for providing valuable information.

\section{References}

1. National Cancer Information Center. Cancer statistics [Internet]. Goyang, Korea: National Cancer Information Center; c2019 [cited 2019 Dec 15]. Available from: https://www.cancer. go.kr/lay1/S1T639C642/contents.do.

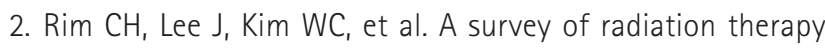
utilization in Korea from 2010 to 2016: focusing on use of intensity-modulated radiation therapy. J Korean Med Sci 2018;33(9):e67.

3. Seo YS, Kim MS, Kang JK, et al. The clinical utilization of radiation therapy in Korea between 2011 and 2015. Cancer Res Treat 2018;50:345-55.

4. Park SW, Oh DH, Bae HS, Cho BC, Park JH, Han SH. Application of intensity modulated radiation therapy (IMRT) in prostate cancer. J Korean Soc Ther Radiol Oncol 2002;20:68-72.

5. Health and Insurance Review and Assessment Service. Primary intensity modulated radiation therapy [Internet]. Wonju, Korea: Health and Insurance Review and Assessment Service; 
c2015 [cited 2019 Dec 15]. Available from: http://opendata. hira.or.kr/op/opc/olapDiagBhvlnfo.do.

6. Huh SJ, Nishimura T, Park W, Nakamura K. Comparison ofradiotherapy infrastructure between Korea and Japan. Jpn J Clin Oncol 2019;49:1024-8.

7. Cho B. Intensity-modulated radiation therapy: a review with a physics perspective. Radiat Oncol J 2018;36:1-10.

8. Mayles WP. Survey of the availability and use of advanced radiotherapy technology in the UK. Clin Oncol 2010;22:63642.

9. Hahn C, Kavanagh B, Bhatnagar A, et al. Choosing wisely: the American Society for Radiation Oncology's top 5 list. Pract Radiat Oncol 2014;4:349-55.

10. Smith BD, Bellon JR, Blitzblau R, et al. Radiation therapy for the whole breast: Executive summary of an American Society for Radiation Oncology (ASTRO) evidence-based guideline. Pract Radiat Oncol 2018;8(3):145-52.

11. Shumway DA, Griffith KA, Pierce $\sqcup$, et al. Wide variation in the diffusion of a new technology: practice-based trends in intensity-modulated radiation therapy (IMRT) use in the State of Michigan, with implications for IMRT use nationally. J Oncol Pract 2015;11:e373-9.

12. Japanese Society for Radiation Oncology. 2017 simple structure survey [Internet]. Tokyo, Japan: Japanese Society for Radiation Oncology; 2018 [cited 2019 Dec 12]. Available from: https://www.jastro.or.jp/medicalpersonnel/data_center/cat6/ cat1/post-6.html.

13. The medical fee points list: radiotherapy. Tokyo, Japan: Igakutushin; 2018.

14. Japanese Society for Radiation Oncology. JASTRO Radiotherapy Cases National Registration Project (JROD) 2018 Survey Report [Internet]. Tokyo, Japan: Japanese Society for Radiation Oncology; 2019 [cited 2019 Dec 12]. Available from: https://www.jastro.or.jp/medicalpersonnel/data_center/cat6/ jrod/2018.html.

15. Huh SJ; Korean Society of Therapeutic Radiology and Oncology (KOSTRO). Current status of the infrastructure and characteristics of radiation oncology in Korea. Jpn J Clin Oncol 2007;37:623-7. 